\title{
Phonon-mediated superconductivity in graphene by lithium deposition
}

\author{
Gianni Profeta ${ }^{1,2 \star}$, Matteo Calandra ${ }^{3}$ and Francesco Mauri ${ }^{3}$
}

Graphene $^{1}$ is the physical realization of many fundamental concepts and phenomena in solid-state physics ${ }^{2}$. However, in the list of graphene's many remarkable properties ${ }^{3-6}$, superconductivity is notably absent. If it were possible to find a way to induce superconductivity, it could improve the performance and enable more efficient integration of a variety of promising device concepts including nanoscale superconducting quantum interference devices, single-electron superconductor-quantum dot devices ${ }^{7,8}$, nanometre-scale superconducting transistors ${ }^{9}$ and cryogenic solid-state coolers ${ }^{10}$. To this end, we explore the possibility of inducing superconductivity in a graphene sheet by doping its surface with alkaline metal adatoms, in a manner analogous to which superconductivity is induced in graphite intercalated compounds ${ }^{11,12}$ (GICs). As for GICs, we find that the electrical characteristics of graphene are sensitive to the species of adatom used. However, contrary to what happens in GICs, Li-covered graphene is superconducting at a much higher temperature with respect to $\mathrm{Ca}$-covered graphene.

As graphene itself is not superconducting, phonon-mediated superconductivity must be induced by an enhancement of the electron-phonon coupling $(\lambda)$,

$$
\lambda=\frac{N(0) D^{2}}{M \omega_{\mathrm{ph}}^{2}}
$$

In equation (1), $N(0)$ is the electronic density of states per spin (DOS) at the Fermi level, $D$ is the deformation potential, and $M$ and $\omega_{\text {ph }}$ are the effective atomic mass and phonon frequency that in metallic alloys reflect the role of the different atomic species and phonon vibrations involved in superconductivity. In undoped graphene $\lambda$ is small and phonon-mediated superconductivity does not occur as the small number of carriers, intrinsic to a semimetal, leads to a vanishingly small $N(0)$. In this respect the situation is similar to the bulk graphite case, where, without intercalation of foreign atoms superconductivity is not stabilized.

A first guess to induce superconductivity could then be to fill by rigid-band doping the carbon $\pi$-states to have enough carriers. However, besides the fact that the $\pi$-DOS grows very slowly with doping, its impact will be hindered by two major difficulties. First, even if the deformation potential related to coupling between $\pi$-bands and in-plane phonon vibrations is large and leads to Kohn anomalies ${ }^{13}$, these vibrations are highly energetic $\left(\omega_{\mathrm{ph}} \approx 0.16 \mathrm{eV}\right)$ and $\lambda$ is small owing to the $\omega_{\mathrm{ph}}^{2}$ factor in the denominator. Second, symmetry forbids the coupling between $\pi$-states and the softer out-of-planes vibrations.

To promote coupling to carbon out-of-plane vibrations, it is then necessary to promote new electronic states at the Fermi level as happens in GICs. Indeed, in superconducting GICs, an intercalant band (interlayer state) occurs at the Fermi

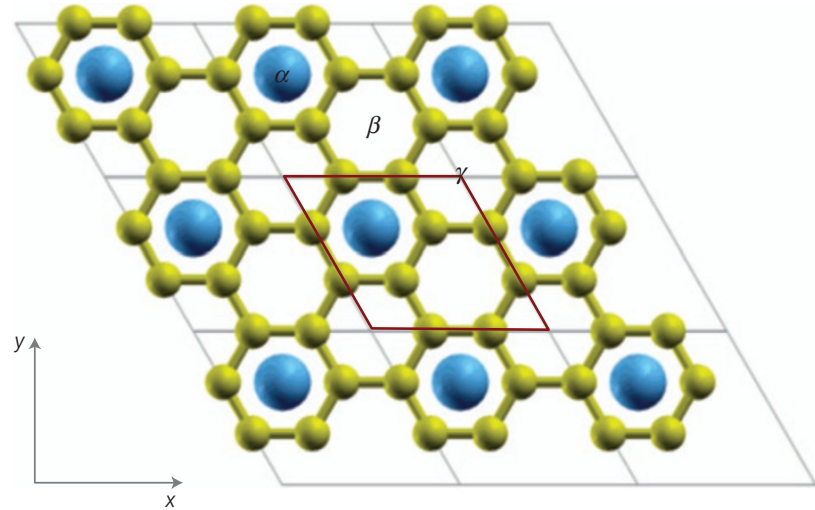

Figure 1 | Crystal structure of metal-adatom-covered graphene. Yellow (blue) spheres represent carbon (metal) atoms. The adatoms sit in the hollow sites of the graphene layer. The bulk $\mathrm{LiC}_{6}$ crystal structure is obtained by periodical repetition of the monolayer along the $z$ axis. The $\mathrm{CaC}_{6}$ bulk phase has a unit cell with three graphene layers along the $z$ axis. For each graphene layer, the calcium atoms occupy different hollow sites of the three allowed in the in-plane unit cell.

Table 1 | Physical parameters of the studied systems.

\begin{tabular}{|c|c|c|c|c|c|c|}
\hline & & $a$ & $h$ & $\lambda$ & $\omega_{\log }$ & $T_{\mathrm{c}}$ \\
\hline $\mathrm{CaC}_{6}$ & Bulk & 4.30 & 2.19 & 0.68 & 284.3 & 11.5 \\
\hline $\mathrm{CaC}_{6}$ & Mono & 4.26 & 2.24 & 0.40 & 309.9 & 1.4 \\
\hline $\mathrm{LiC}_{6}$ & Bulk & 4.29 & 1.83 & 0.33 & 715.7 & 0.9 \\
\hline $\mathrm{LiC}_{6}$ & Mono & 4.26 & 1.83 & 0.61 & 277.8 & 8.1 \\
\hline
\end{tabular}

Optimized structural parameters (lattice constant $(a)$ and adatom-graphene distance $(h)$ in $\AA$ ), electron-phonon coupling $(\lambda)$, logarithmic frequency average $\left(\omega_{\log }\right.$ in $\left.\mathrm{cm}^{-1}\right)$ and superconducting critical temperature $\left(T_{\mathrm{C}}\right.$ in Kelvin) for $\mathrm{CaC}_{6}$ and $\mathrm{LiC}_{6}$ systems.

level ${ }^{14,15}$ and has multiple beneficial effects on $\lambda$ (ref. 20): the number of carriers is enhanced; coupling to carbon out-of-plane vibrations is promoted; and coupling to intercalant vibrations occurs with a corresponding enhancement of the deformation potential and a reduction of the effective $M \omega_{\mathrm{ph}}^{2}$ term in the denominator of equation (1).

In GICs, not all types of intercalant atom leading to an interlayer state are equally effective in increasing $T_{\mathrm{c}}$ (ref. 15). The larger $T_{\mathrm{c}}$ is indeed obtained when the distance $(h)$ between the intercalant atom and the graphite plane is smaller. The reason for this is that the closer the intercalant electrons are to the planes, the larger are the deformation potentials, $D$, of the intercalant and carbon out-of-plane modes ${ }^{15,21}$. This is demonstrated by the increase in

${ }^{1}$ Dipartimento di Fisica Università degli Studi di L'Aquila and SPIN-CNR, I-67100 L'Aquila, Italy, ${ }^{2}$ Max-Planck Institute of Microstructure Physics, Weinberg 2, D-06120 Halle, Germany, ${ }^{3}$ IMPMC, Universitè Paris 6, CNRS, 4 PI. Jussieu, 75015 Paris, France. *e-mail: gianni.profeta@aquila.infn.it. 

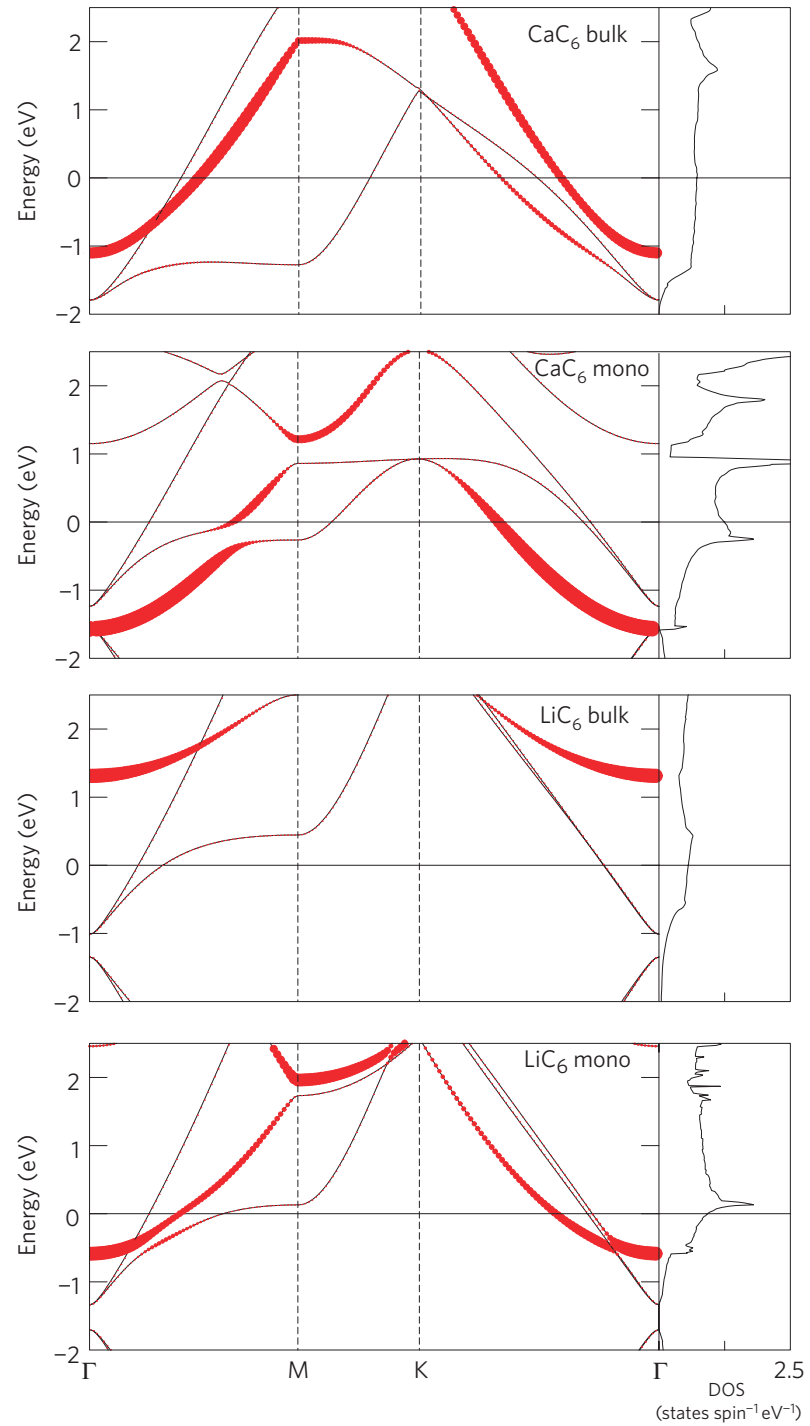

Figure 2 | Electronic band structure. From top to bottom: band structure and total density of states per spin (DOS) of $\mathrm{CaC}_{6}$ bulk (plotted along the high-symmetry lines of the monolayer lattice), $\mathrm{CaC}_{6}$ monolayer, $\mathrm{LiC}_{6}$ bulk and $\mathrm{LiC}_{6}$ monolayer. The Fermi energy is set to zero. The Dirac point is folded at the $\Gamma$-point at $-1.56 \mathrm{eV}$ from the Fermi level in the $\mathrm{LiC}_{6}$ monolayer and at $-1.44 \mathrm{eV}$ in the $\mathrm{CaC}_{6}$ monolayer. A small gap is opened by the metal-intercalated graphene superstructure. The thickness of the bands is proportional to the intercalate/adatom $s$ character.

$T_{\mathrm{c}}$ from $\mathrm{BaC}_{6}$ (not superconducting) to $\mathrm{SrC}_{6}\left(T_{\mathrm{c}}=1.65 \mathrm{~K}\right.$; ref. 16$)$ and finally to $\mathrm{CaC}_{6}\left(T_{\mathrm{c}}=11.5 \mathrm{~K}\right.$; refs 11,12$)$, which correlates with the distance between the intercalant and the graphene plane $(h)$. For the alkalis and alkaline earths considered, these distances are $h\left(\mathrm{BaC}_{6}\right)=2.62 \AA, h\left(\mathrm{SrC}_{6}\right)=2.47 \AA$ and $h\left(\mathrm{CaC}_{6}\right)=2.26 \AA($ ref. 15$)$. The increase in the $\mathrm{CaC}_{6}$ critical temperature under hydrostatic pressure $^{17}$ is a further confirmation of this trend.

However, a too small intercalant-graphite layer distance could also be detrimental for superconductivity. Indeed, the quantum confinement of the interlayer state in a too narrow region between the planes could result in an upshift of the intercalant band well above the Fermi energy (see Fig. 4 in ref. 14). In this case, the ionization of the intercalant atom is complete and superconductivity is totally suppressed, as in bulk $\mathrm{LiC}_{6}\left(h\left(\mathrm{LiC}_{6}\right)=1.85 \AA\right)$.

Following these accepted guidelines for GICs, we explore the possibility to induce superconductivity in graphene by foreignatom coverage (Fig. 1), by means of first-principles density
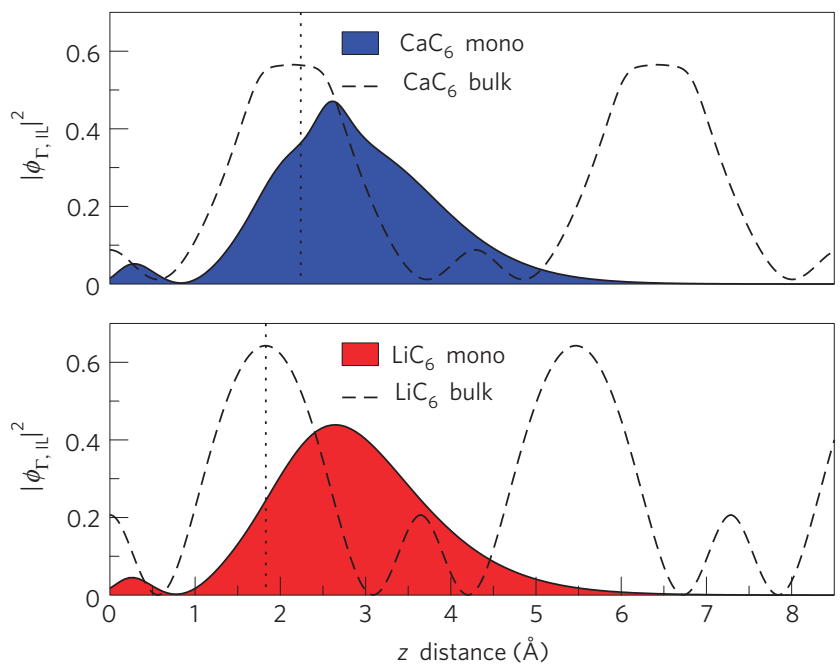

Figure 3 | Interlayer-state wavefunction. Planar (in the $x-y$ direction) average of $\left|\phi_{\Gamma, \mathrm{LL}}\right|^{2}$ along the perpendicular (with respect to the graphene layer) direction ( $z$ ). IL, interlayer. The vertical dotted lines represent the $z$ position of the calcium and lithium adatoms in the monolayer.

functional theory calculations ${ }^{18}$ in the local-density approximation with the parametrization of ref. 19.

The parallel with GICs is tempting, and in particular, considering that calcium-intercalated graphite shows the highest superconducting critical temperature among GICs, the first example we consider is calcium-doped graphene and for comparison we simulated calcium-intercalated graphite. Calcium, as is the case for other alkaline metals, adsorbs in the hollow sites of graphene (Fig. 1 and Table 1).

The electronic band structure of both compounds is reported in Fig. 2. As in the bulk, the deposition of calcium on top of graphene leads to an interlayer band at the Fermi level. In passing from the bulk to the monolayer system, the removal of the quantum confinement for the electrons along the $c$ direction lowers the energy of the interlayer state. The interlayer will then be more occupied and the carbon $\pi$-bands less doped.

However, the presence of the interlayer state at the Fermi level alone is not sufficient to guarantee large electron-phonon coupling. Indeed, a sizeable coupling between electrons and outof-plane carbon vibrations is needed. Such coupling is due to transitions between the interlayer band and the $\pi^{*}$ states $^{20}$. As the localization of the interlayer state as close as possible to the graphene layer enhances the coupling ${ }^{14,20,21}$, in Fig. 3 we compare the planar average of the interlayer charge density (calculated at the $\Gamma$-point of the Brillouin zone) in the case of bulk and monolayer $\mathrm{CaC}_{6}$. In the monolayer, the interlayer charge density spills out in the vacuum region, whereas in the bulk case, it is much more confined between the graphene and adatom layers (it has a period of $2 h$ ).

On the basis of this last consideration, we can infer that the superconducting critical temperature of the $\mathrm{CaC}_{6}$ monolayer should be lower than its bulk counterpart. To verify this hypothesis, we calculated the vibrational spectrum and the electron-phonon coupling $\lambda=2 \int \mathrm{d} \omega \alpha^{2} F(\omega) / \omega$ of both compounds. The results are summarized in Figs 4 and 5 and Table 1. First we notice that the monolayer system is dynamically stable, not showing a tendency to displacive instabilities, with a phonon dispersion characterized by three regions: the low-energy region of adatom-related modes (up to $300 \mathrm{~cm}^{-1}$ ) extending up to $400 \mathrm{~cm}^{-1}$ when mixed with carbon out-of-plane modes $\left(C_{z}\right)$, an intermediate region of $C_{z}$ modes $\left(400-900 \mathrm{~cm}^{-1}\right)$ and the high-energy region characterized by $\mathrm{C}-\mathrm{C}$ stretching modes. 

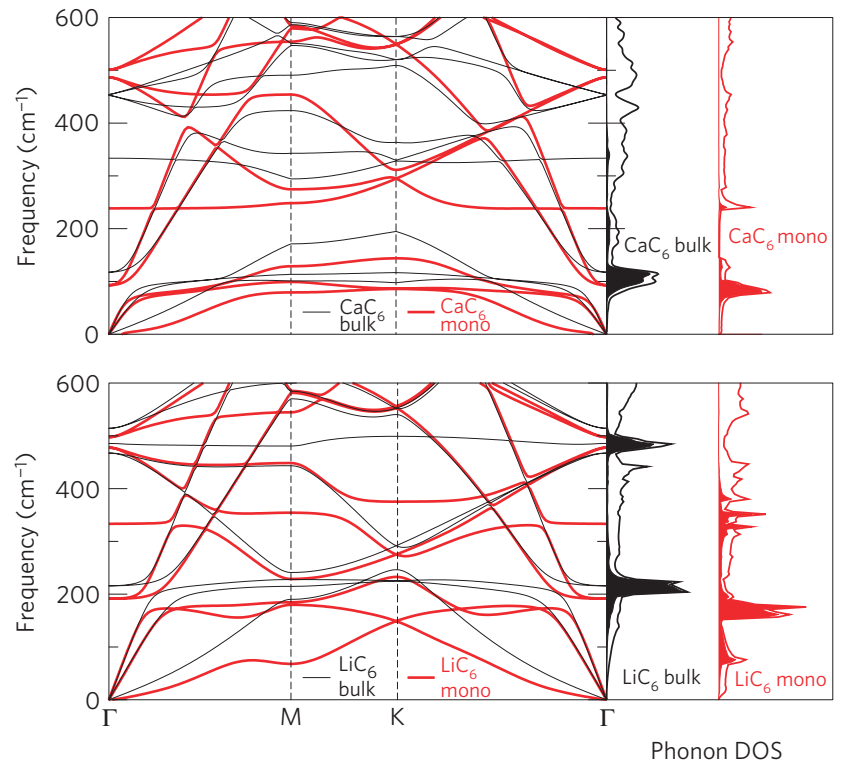

Figure 4 | Dynamical properties. Phonon frequency dispersion and phonon density of states of $\mathrm{CaC}_{6}$ (top panel) and $\mathrm{LiC}_{6}$ (bottom panel). Bulk (black) and monolayer (red) cases are considered through resolving the adatom (shaded) contribution to the total (solid line) phonon DOS. The phonon dispersion in the full energy range is reported in the Supplementary Information.

The main difference between bulk and monolayer phonons is the softening of calcium vibrations. From inspection of the $\alpha^{2} F(\omega)$ (which gives the contribution of each frequency to the total electron-phonon coupling), we note the low contribution of the $C_{z}$ modes (around $500 \mathrm{~cm}^{-1}$ ) to the total electron-phonon coupling in the monolayer case with respect to the bulk $\mathrm{CaC}_{6}$, as expected from the above considerations. Whereas the critical temperature of the bulk compound is $11.5 \mathrm{~K}$ (see Methods), in the monolayer case it decreases to $1.4 \mathrm{~K}$.

This scenario indicates that, as a general rule, the removal of quantum confinement should be detrimental for the electronphonon coupling when compared with the same bulk (threedimensional periodic) system, owing to the shift in the interlayer wavefunction away from the graphene layer. Every metal-covered graphene should have (at least in the same stoichiometry) a reduced superconducting critical temperature with respect to the corresponding GIC.

However, there is at least one example among GICs (to the best of our knowledge) that should be further explored, namely the stage- 1 lithium-intercalated compound, $\mathrm{LiC}_{6}$ (ref. 22). In bulk $\mathrm{LiC}_{6}$, the interlayer state is completely empty (see Fig. 2), as the strong confinement along the $z$ direction (Fig. 3) prevents its occupation. For this reason $\mathrm{LiC}_{6}$ is not superconducting. On going from $\mathrm{LiC}_{6}$ bulk to a $\mathrm{LiC}_{6}$ monolayer, the removal of quantum confinement along the $c$ direction (Fig. 3) could bring the interlayer to the Fermi level. This is confirmed by the calculated band structure (Fig. 2). Figure 3 shows that the spatial extension of the interlayer for lithium is the same as in calcium, but as the interlayer is strongly localized around the adatom and closer to the graphene layer, we can expect an enhancement of the total electron-phonon coupling.

The phonon spectrum confirms, even in this case, the dynamical stability of the monolayer system and the comparison with the bulk counterpart reveals that low-energy adatom modes and carbon vibrations in the direction perpendicular to the plane are strongly softened in the monolayer. This indicates that the first two phonon branches, related to the in-plane displacements of lithium atoms,
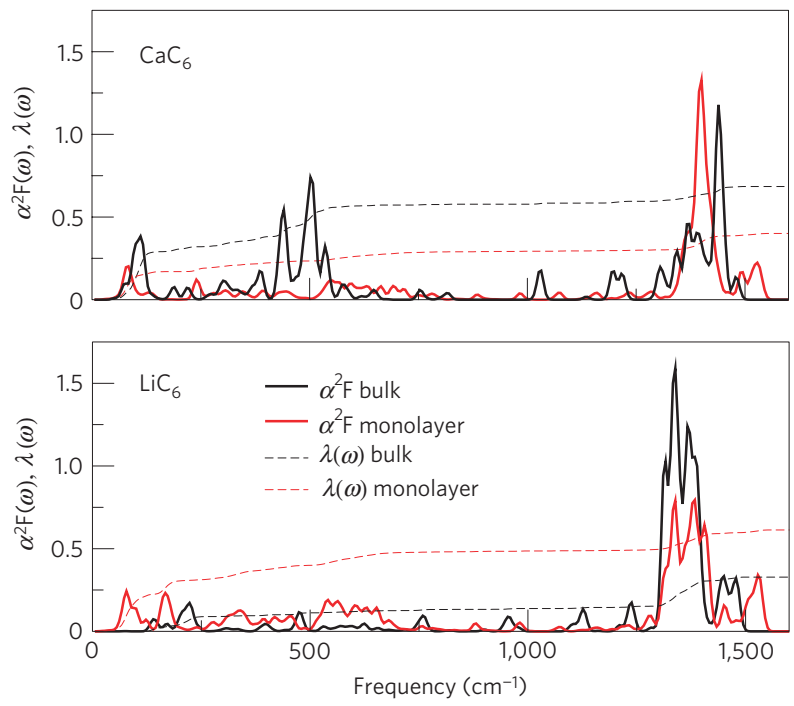

Figure 5 | Eliashberg function. Bulk and monolayer $\mathrm{CaC}_{6}$ (top panel) and $\mathrm{LiC}_{6}$ (bottom panel) $\alpha^{2} F(\omega) . \lambda(\omega)$ is also reported in the same graphs.

and the un-dispersing Einstein mode displacing lithium and carbon atoms out-of-phase along the $z$ direction at around $500 \mathrm{~cm}^{-1}$, should undergo enhanced electron-phonon coupling with respect to the bulk. On the contrary, in the $\mathrm{CaC}_{6}$ monolayer, adatom vibration and carbon vibrations along $z$ are essentially at the same energy as in bulk $\mathrm{CaC}_{6}$.

We found that bulk $\mathrm{LiC}_{6}$ is a weak electron-phonon coupling $\lambda=0.33$ ( 0.18 from carbon in-plane vibrations and 0.1 from intercalant vibrations) superconductor with an estimated superconducting critical temperature $T_{\mathrm{c}}=0.9 \mathrm{~K}$, theoretically confirming the absence of superconductivity in $\mathrm{LiC}_{6}$ above $1 \mathrm{~K}$. In the case of monolayer $\mathrm{LiC}_{6}$, the total electron-phonon coupling is $\lambda=0.61$, with a superconducting critical temperature up to $8.1 \mathrm{~K}$. The main contributions to the electron-phonon coupling come from the lowenergy lithium modes and carbon vibrations along $z$, as expected from the above considerations, with an appreciable contribution from $\mathrm{C}-\mathrm{C}$ stretching modes $(0.1)$.

Thus, we find that to induce superconductivity in graphene, it is beneficial to bring the interlayer state to the Fermi energy and to localize it as close as possible to the graphene plane. In the case of $\mathrm{a} \mathrm{LiC}_{6}$ monolayer, the presence of the interlayer state switches on the electron-phonon coupling of $C_{z}$ modes that is inactive in the bulk, and increases the contribution of intercalant modes. Graphene can be made superconducting by the deposition of lithium atoms on top of it.

As lithium readily intercalates into graphite even at $100 \mathrm{~K}$ (ref. 23), it is possible to incorporate lithium atoms even below the graphene sheet. This possibility, as shown by our calculations, is indeed favourable with respect to the development of a superconducting phase. In fact, the double adsorption should double the presence of the interlayer state at the Fermi level (one coming from each side of graphene) as we indeed verified.

$\mathrm{Li}_{2} \mathrm{C}_{6}$ shows an increased coupling in all of the frequency range owing to the additional strongly coupled interlayer bands, with an electron-phonon coupling of 1.0 giving rise to a critical temperature of around 17-18 K (see Supplementary Information).

We note again that bulk $\mathrm{LiC}_{6}$ is a candidate material to show a remarkable metal-to-superconductor transition when exfoliated to one layer. The reason is that in single-layer $\mathrm{LiC}_{6}$ the interlayer band crosses the Fermi level, enhances the electron-phonon coupling and can induce superconductivity. Our work demonstrates that superconducting properties of adatoms on graphene are quite different from their bulk GIC counterparts. 


\section{Methods}

The results reported here were obtained from first-principles density functional theory in the local density approximation ${ }^{19}$. The QUANTUM-ESPRESSO (ref. 18) package was used with norm-conserving pseudopotentials and a plane-wave cutoff energy of $65 \mathrm{Ry}$. All of the structures considered were relaxed to their minimum energy configuration following the internal forces on atoms and the stress tensor of the unit cell.

The monolayer systems were simulated in the $\sqrt{3} \times \sqrt{3} R 30^{\circ}$ in-plane unit cell (with respect to a standard graphene lattice, see Fig. 1) with one adatom per unit cell. Phonon frequencies were calculated using the linear-response technique on a phonon wave-vector mesh of $12 \times 12$ with a $14 \times 14$ uniform electron-momentum grid. The electron-phonon coupling parameter was calculated with electron momentum $k$-mesh up to $40 \times 40$.

The $\mathrm{CaC}_{6}$ bulk compound was simulated in the experimentally found structure with $\alpha \beta \gamma$ stacking ${ }^{11}$ with a uniform electron-momentum $k$-mesh integration of $8 \times 8 \times 8$. The phonon frequencies were calculated on a $4 \times 4 \times 4$ phonon-momentum mesh and the electron-phonon coupling was integrated on a $20 \times 20 \times 20$ electron-momentum mesh.

Bulk $\mathrm{LiC}_{6}$ has an $\alpha \alpha$ stacking and was simulated with an electron-momentum mesh of $12 \times 12 \times 10$ and a $6 \times 6 \times 6$ phonon-momentum grid for the calculation of phonon frequencies. A $30 \times 30 \times 25$ electron-momentum mesh was used for the electron-phonon coupling.

The Eliashberg function $\alpha^{2} F(\omega)$ is defined as:

$$
\begin{aligned}
\alpha^{2} F(\omega)= & \frac{1}{N(0) N_{k} N_{q}} \sum_{n \mathbf{k}, m \mathbf{q}, v}\left|g_{n \mathbf{k}, m \mathbf{k}+\mathbf{q}}^{v}\right|^{2} \\
& \times \delta\left(\varepsilon_{n \mathbf{k}}\right) \delta\left(\varepsilon_{m \mathbf{k}+\mathbf{q}}\right) \delta\left(\omega-\omega_{\mathbf{q}}^{v}\right)
\end{aligned}
$$

where $N(0)$ is the total density of states per spin and $N_{k}$ and $N_{q}$ the total numbers of $k$ and $q$ points, respectively. The electron eigenvalues are labelled with the band index $(n$ and $m)$ and the wavevector $(\mathbf{k}$ and $\mathbf{k}+\mathbf{q})$, while phonon frequencies with the mode number $(v)$ and the wavevector $(\mathbf{q}) \cdot g_{n \mathbf{k}, m \mathbf{k}+\mathbf{q}}^{v}$ represents the electron-phonon matrix element. The total electron-phonon coupling $\lambda(\omega)$ plotted in Fig. 5 is defined as:

$$
\lambda(\omega)=2 \int_{0}^{\omega} \mathrm{d} \omega^{\prime} \frac{\alpha^{2} F\left(\omega^{\prime}\right)}{\omega^{\prime}}
$$

The total electron-phonon coupling is $\lambda(\omega \rightarrow \infty)$. The superconducting critical temperature was estimated using the Allen-Dynes formula with $\mu^{*}=0.115$, which fits the experimental critical temperature measured in $\mathrm{CaC}_{6} \mathrm{GIC}$ (ref. 11) As the doping levels considered in the present work are fairly large, the values of $T_{\mathrm{c}}$ should be weakly renormalized by the phase fluctuations of the Kosterlitz and Thouless transition ${ }^{24}$.

Received 28 April 2011; accepted 23 November 2011; published online 10 January 2012

\section{References}

1. Novoselov, K. S. et al. Two-dimensional atomic crystals. Proc. Natl Acad. Sci. USA 102, 10451-10453 (2005).

2. Geim, A. K. Graphene: Status and prospects. Science 324, 1530-1534 (2009).

3. Katsnelson, M. I., Novoselov, K. S. \& Geim, A. K. Chiral tunnelling and the Klein paradox in graphene. Nature Phys. 2, 620-625 (2006).

4. Novoselov, K. S. et al. Room-temperature quantum Hall effect in graphene. Science 315, 1379 (2007).

5. Zhang, Y., Tan, Y. W., Stormer, H. L. \& Kim, P. Experimental observation of the quantum Hall effect and Berry's phase in graphene. Nature $\mathbf{4 3 8}$ 201-204 (2005).
6. Nair, R. R. et al. Fine structure constant defines visual transparency of graphene. Science 320, 1308 (2008).

7. De Franceschi, S., Kouwenhoven, L., Schönenberger, Ch. \& Wernsdorfer, W. Hybrid superconductor-quantum dot devices. Nature Nanotech. 5, 703711 (2010).

8. Huefner, M. et al. Scanning gate microscopy measurements on a superconducting single-electron transistor. Phys. Rev. B 79, 134530 (2009).

9. Delahaye, J. et al. Low-noise current amplifier based on mesoscopic Josephson junction. Science 299, 1045-1048 (2003).

10. Olli-Pentti, S. et al. Heat-transistor: Demonstration of gate-controlled electronic refrigeration. Phys. Rev. Lett. 99, 027203 (2007).

11. Emery, N. et al. Superconductivity of bulk $\mathrm{CaC}_{6}$. Phys. Rev. Lett. 95, 087003 (2005).

12. Weller, T. et al. Superconductivity in the intercalated graphite compounds $\mathrm{C}_{6} \mathrm{Yb}$ and $\mathrm{C}_{6} \mathrm{Ca}$. Nature Phys. 1, 39-41 (2005).

13. Piscanec, S. et al. Kohn anomalies and electron-phonon interactions in graphite. Phys. Rev. Lett. 93, 185503 (2004).

14. Csányi, G. et al. The role of the interlayer state in the electronic structure of superconducting graphite intercalated compounds. Nature Phys. 1, 42-45 (2005).

15. Calandra, M. \& Mauri, F. Possibility of superconductivity in graphite intercalated with alkaline earths investigated with density functional theory. Phys. Rev. B 74, 094507 (2006).

16. Kim, J. S. et al. Superconductivity in heavy alkaline-earth intercalated graphites. Phys. Rev. Lett. 99, 027001 (2007).

17. Gauzzi, A. et al. Enhancement of superconductivity and evidence of structural instability in intercalated graphite $\mathrm{CaC}_{6}$ under high pressure. Phys. Rev. Lett. 98, 067002 (2007).

18. Giannozzi, P. et al. QUANTUM ESPRESSO: A modular and open-source software project for quantum simulations of materials. J. Phys. Condens. Matter 21, 395502 (2009).

19. Appendix C of J. P. Perdew and A. Zunger, Self-interaction correction to density-functional approximations for many-electron systems, Phys. Rev. B 23, 5048-5079 (1981).

20. Calandra, M. \& Mauri, F. Theoretical explanation of superconductivity in $\mathrm{C}_{6}$ Ca. Phys. Rev. Lett. 95, 237002 (2005).

21. Boeri, L. et al. Electron-phonon interaction in graphite intercalation compounds. Phys. Rev. B 76, 064510 (2007).

22. Guerarda, D. \& Herold, A. Intercalation of lithium into graphite and other carbons. Carbon 13, 337-345 (1975).

23. Caragiu, M. \& Finberg, S. Alkali metal adsorption on graphite: A review. J. Phys. Condens. Matter 17, R995-R1024 (2005).

24. Loktev, V. M. \& Turkowski, V. Possible high-temperature superconductivity in multilayer graphane: Can the cuprates be beaten? J. Low Temp. Phys. 164, 264-271 (2011).

\section{Acknowledgements}

This work was supported by a CINECA-HPC ISCRA grant, the EU DEISA-SUPERMAG project and an HPC grant at CASPUR. Some of the calculations were carried out at the IDRIS supercomputing centre (project 91202).

\section{Author contributions}

All of the authors contributed equally to the project.

\section{Additional information}

The authors declare no competing financial interests. Supplementary information accompanies this paper on www.nature.com/naturephysics. Reprints and permissions information is available online at http://www.nature.com/reprints. Correspondence and requests for materials should be addressed to G.P. 